\title{
Probing Heterogeneous Efflorescence of Mars Relevant Salts with an Optical Levitator
}

Shuichi B. Ushijima ${ }^{1,2}$, Raina V. Gough ${ }^{1,2}$, Margaret A. Tolbert ${ }^{1,2, *}$

${ }^{1}$ Department of Chemistry University of Colorado Boulder, 215 UCB Boulder, CO, 80309

${ }^{2}$ Cooperative Institute for Research in Environmental Science, 216 UCB Boulder, CO, 80309

*Corresponding Author e-mail: margaret.tolbert @ colorado.edu phone: (303) 492-3179 

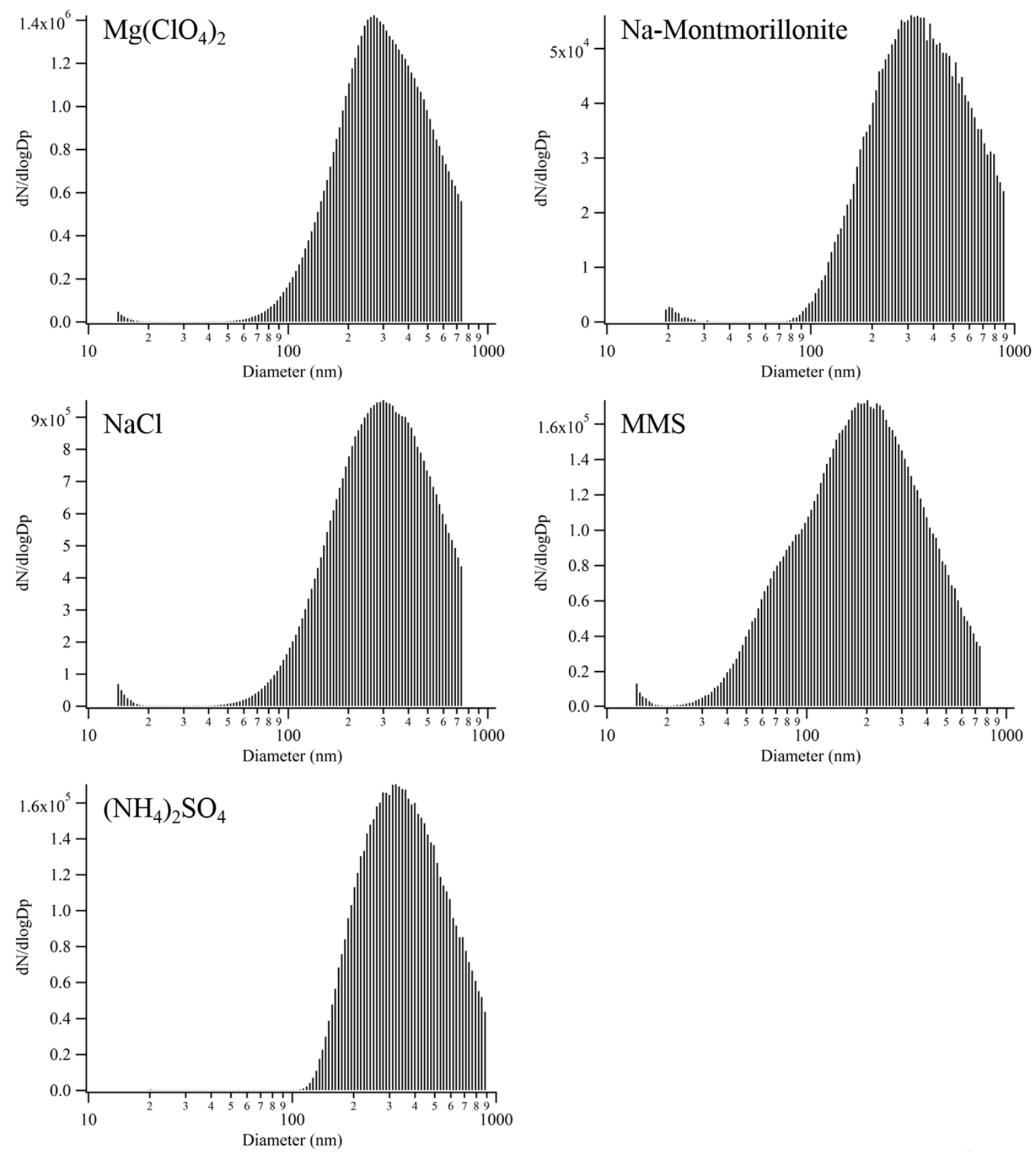

Figure S1: Particle size distribution of $\mathrm{Mg}\left(\mathrm{ClO}_{4}\right)_{2}, \mathrm{NaCl},\left(\mathrm{NH}_{4}\right)_{2} \mathrm{SO}_{4}, \mathrm{Na}$-montmorillonite ${ }^{1}$, and MMS particles made with the nebulizer. Distributions are the average of 3 samples taken in an SMPS. The high end cutoff of particle size is due to the upper limit of the instrument and not due to the actual distribution. 


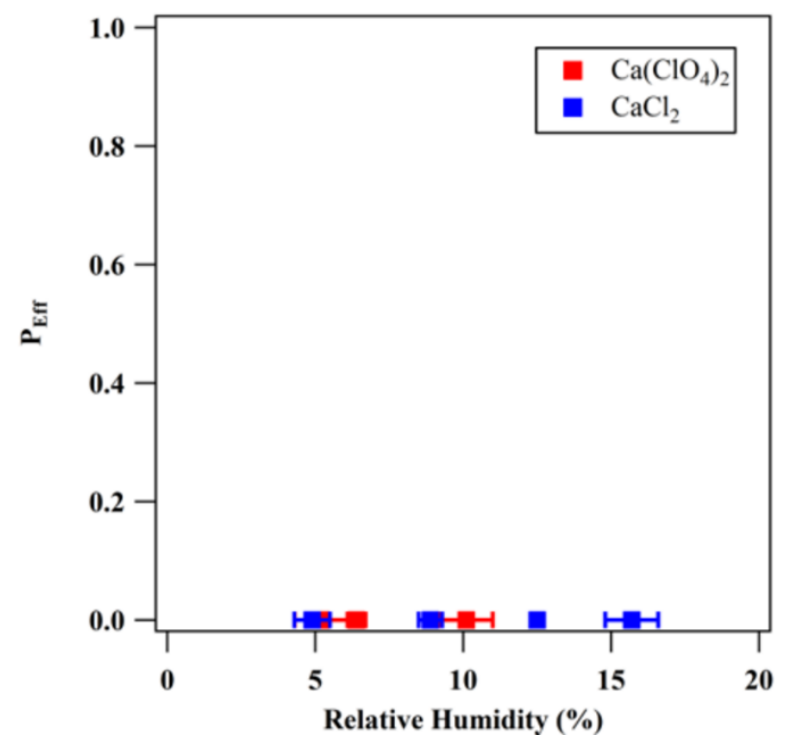

Figure S2 Contact efflorescence experiments for $\mathrm{Ca}\left(\mathrm{ClO}_{4}\right)_{2}$ and $\mathrm{CaCl}_{2}$ by Na-montmorillonite.

Table S1: Lattice constants and crystal system

\begin{tabular}{|c|c|c|c|c|c|}
\hline & \multicolumn{3}{|c|}{ Lattice Constants } & \multirow[b]{2}{*}{ Crystal System } & \multirow[b]{2}{*}{ Reference } \\
\hline & $\mathrm{a}$ & $\mathrm{b}$ & $\mathrm{c}$ & & \\
\hline $\mathrm{Mg}\left(\mathrm{ClO}_{4}\right)_{2} \bullet 6 \mathrm{H}_{2} \mathrm{O}$ & 7.775 & 13.494 & 5.27 & Orthorhombic & 2 \\
\hline $\mathrm{NaCl}$ & 5.64 & 5.64 & 5.64 & Cubic & 3 \\
\hline$\left(\mathrm{NH}_{4}\right)_{2} \mathrm{SO}_{4}$ & 7.73 & 10.56 & 5.95 & Orthorhombic & 3 \\
\hline Na-Montmorillonite & 5.17 & 8.94 & 9.95 & Monoclinic & 3 \\
\hline $\mathrm{MgCl}_{2} \bullet 4 \mathrm{H}_{2} \mathrm{O}$ & 7.26 & 8.43 & 11.04 & Orthorhombic & 4 \\
\hline $\mathrm{Ca}\left(\mathrm{ClO}_{4}\right)_{2} \bullet 4 \mathrm{H}_{2} \mathrm{O}$ & 5.49 & 7.85 & 11.57 & Triclinic & 5 \\
\hline $\mathrm{CaCl}_{2} \bullet 6 \mathrm{H}_{2} \mathrm{O}$ & 7.88 & 7.88 & 3.95 & Hexagonal & 3 \\
\hline Olivine & 4.779 & 10.277 & 5.995 & Orthorhombic & 3 \\
\hline Orthopyroxene & 18.316 & 8.907 & 5.218 & Orthorhombic & 3 \\
\hline Clinopyroxene & 9.794 & 8.906 & 5.319 & Monoclinic & 3 \\
\hline Feldspar (Orthoclase) & 8.56 & 12.96 & 7.299 & Monoclinic & 3 \\
\hline Hematite & 5.038 & 5.038 & 13.772 & Hexagonal & 3 \\
\hline Nontronite & 5.277 & 9.14 & 9.78 & Monoclinic & 3 \\
\hline Kaolinite & 5.13 & 8.89 & 7.25 & Triclinic & 3 \\
\hline
\end{tabular}




\begin{tabular}{|c|c|c|c|c|c|}
\hline Chlorite & 5.3363 & 9.24 & 14.37 & Monoclinic & 3 \\
\hline Illite & 5.18 & 8.98 & 10.32 & Monoclinic & 3 \\
\hline Prehnite & 4.646 & 5.483 & 18.486 & Orthorhombic & 3 \\
\hline Quartz & 4.9133 & 4.9133 & 5.4053 & Hexagonal & 3 \\
\hline $\mathrm{MgCO} 3$ (Magnesite) & 4.633 & 4.633 & 15.016 & Rhombohedral & 3 \\
\hline $\mathrm{CaCO} 3$ (Calcite) & 4.99 & 4.99 & 17.0615 & Rhombohedral & 3 \\
\hline $\mathrm{MgSO}_{\text {(Kieserite) }}$ & 6.891 & 7.624 & 7.645 & Monoclinic & 3 \\
\hline $\mathrm{CaSO}_{4}$ (Gypsum) & 5.674 & 15.1049 & 6.4909 & Monoclinic & 3 \\
\hline
\end{tabular}

\section{References}

1. Ushijima, S. B.; Davis, R. D.; Tolbert, M. A. Immersion and Contact Efflorescence Induced by Mineral Dust Particles. J. Phys. Chem. A, 2018, 122, 1303 - 1311.

2. Robertson, K.; Bish, D. Stability of phases in the $\mathrm{Mg}\left(\mathrm{ClO}_{4}\right)_{2} \cdot n \mathrm{H}_{2} \mathrm{O}$ system and implications for perchlorate occurrences on Mars. J. Geophys. Res., 2011, 116, E07006.

3. Downs, R. T.; Hall-Wallace, M. The American Mineralogist crystal structure database. Am. Mineral., 2003, 88, 247-250.

4. Schmidt H.; Hennings, E.; Voigt, W. Magnesium chloride tetrahydrate, $\mathrm{MgCl}_{2} \cdot 4 \mathrm{H}_{2} \mathrm{O}$. Acta Cryst., 2012, C68, i4-i6.

5. Hennings, E.; Schmidt, H.; Voigt, W. Crystal structures of $\mathrm{Ca}\left(\mathrm{ClO}_{4}\right)_{2} \cdot 4 \mathrm{H}_{2} \mathrm{O}$ and $\mathrm{Ca}\left(\mathrm{ClO}_{4}\right)_{2} \cdot 6 \mathrm{H}_{2} \mathrm{O}$. Acta Cryst., 2014, E70, 489-493. 